\title{
Minimal fixed point sets of relative maps
}

\author{
by
}

\author{
Xuezhi Z h a o (Beijing)
}

\begin{abstract}
Let $f:(X, A) \rightarrow(X, A)$ be a self map of a pair of compact polyhedra. It is known that $f$ has at least $N(f ; X, A)$ fixed points on $X$. We give a sufficient and necessary condition for a finite set $P(|P|=N(f ; X, A))$ to be the fixed point set of a map in the relative homotopy class of the given map $f$. As an application, a new lower bound for the number of fixed points of $f$ on $\mathrm{Cl}(X-A)$ is given.
\end{abstract}

1. Introduction. Nielsen fixed point theory is concerned with the determination of minimal fixed point sets of maps (see [1] or [4] for introduction). The Nielsen number $N(f)$ provides a homotopy invariant lower bound for the number of fixed points of a map $f: X \rightarrow X$, which can be realized in fairly general cases. More precisely, a space $X$ is said to be a Nielsen space if every map $f: X \rightarrow X$ is homotopic to a map $g: X \rightarrow X$ which has $N(f)$ fixed points and if these fixed points can lie anywhere in $X$. It is known that a compact polyhedron $X$ will be a Nielsen space if either $X$ has no local cut point and is not a surface with negative Euler characteristic, or $X$ is contractible, or $X$ is a circle $S^{1}$ (see [3, Main Theorem]).

Relative Nielsen fixed point theory provides information about fixed point sets of relative maps, i.e. maps of the form $f:(X, A) \rightarrow(X, A)$. A relative Nielsen number $N(f ; X, A)$ was introduced in [5]; it is the lower bound of the number of fixed points of maps in the relative homotopy class of $f$. Here, homotopies between relative maps are always relative ones, i.e. maps of the form $H:(X \times I, A \times I) \rightarrow(X, A)$. So, it is natural to ask the following:

Problem 1.1. For $f:(X, A) \rightarrow(X, A)$ and a finite set $P \subset X$ with cardinality $|P|=N(f ; X, A)$, does there exist a map g homotopic to $f$ with fixed point set Fix $g=P$ ?

1991 Mathematics Subject Classification: 55M20, 05 C90.

Key words and phrases: fixed point class, minimal fixed point set, relative Nielsen number, bipartite graph, matching.

Supported by the Natural Science Foundation of Beijing (1992001). 
It is obvious that the answer is "no" if $P$ is an arbitrary set. The restriction $\bar{f}: A \rightarrow A$ of $f$ to $A$ has at least $N(\bar{f})$ fixed points in $A$. From [6] and [8], we know that any map in the homotopy class of $f$ has at least $\widetilde{N}(f ; X, A)$ fixed points in $\mathrm{Cl}(X-A)$ and at least $N(f ; X-A)$ fixed points in $X-A$. Furthermore, if $f$ has a minimal fixed point set, i.e. $\mid$ Fix $f \mid=N(f ; X, A)$, then $f$ has at least $\widetilde{n}(f ; X, A)$ fixed points on $\operatorname{Bd}(A)$. A brief definition of these relative Nielsen numbers will be given in Section 3. The conditions following from the minimal fixed point set assumption are summarized in:

Theorem $1.2([9$, Theorem 4.2]). If $f:(X, A) \rightarrow(X, A)$ has $N(f ; X, A)$ fixed points, then $f$ has:

(1) at most $N(\bar{f})-\widetilde{n}(f ; X, A)$ fixed points in $\operatorname{Int}(A)$,

(2) at least $\widetilde{n}(f ; X, A)$ and at most $N(\bar{f})+N_{1010}(f ; X, A)$ fixed points on $\operatorname{Bd}(A)$,

(3) at least $N(\bar{f})$ and at most $N(\bar{f})+N_{1010}(f ; X, A)$ fixed points in $A$,

(4) at least $N(f ; X-A)$ and at most $N_{1010}(f ; X, A)+N(f ; X-A)$ fixed points in $X-A$,

(5) at least $\tilde{N}(f ; X, A)$ and at most $N(f ; X, A)$ fixed points in $\mathrm{Cl}(X-A)$.

For the realization, we have

Theorem 1.3 ([9, Theorem 4.3]). Let $(X, A)$ be a pair of compact polyhedra such that:

1) $X$ is connected,

2) $X-A$ has no local cut point and is not a 2-manifold,

3) every component of $A$ is a Nielsen space with nonempty interior,

4) $A$ can be by-passed in $X$.

Let $f:(X, A) \rightarrow(X, A)$. If $\widetilde{n}(f ; X, A) \leq k_{1} \leq N(\bar{f})$ and $0 \leq k_{2} \leq$ $N_{1010}(f ; X, A)$, then we can homotope $f$ to a map $g:(X, A) \rightarrow(X, A)$ with $N(f ; X, A)$ fixed points in $X$, of which $N(\bar{f})-k_{1}$ lie in $\operatorname{Int}(A), N(f ; X-A)$ $+k_{2}$ lie in $X-A$, and therefore $k_{1}+N_{1010}(f ; X, A)-k_{2}$ lie on $\operatorname{Bd}(A)$.

Does this theorem mean that, if $(X, A)$ satisfies the conditions of the theorem, then any finite set $P$ with $|P|=N(f ; X, A),|P \cap \operatorname{Int}(A)| \leq N(\bar{f})-$ $\widetilde{n}(f ; X, A)$ and $N(f ; X-A) \leq|P \cap(X-A)| \leq N(f ; X-A)+N_{1010}(f ; X, A)$ can be the fixed point set of a relative map in the homotopy class of the given map $f:(X, A) \rightarrow(X, A)$ ? Unfortunately not. An example is given below:

EXAMPLE 1.4. Let $X$ be a solid torus in Euclidean space $\mathbb{R}^{3}$, which is constructed by rotating the 2 -disc $\left\{\left(x_{1}, x_{2}, 0\right) \mid x_{1}^{2}+\left(x_{2}-3\right)^{2} \leq 4\right\}$ around the $x_{1}$-axis. 


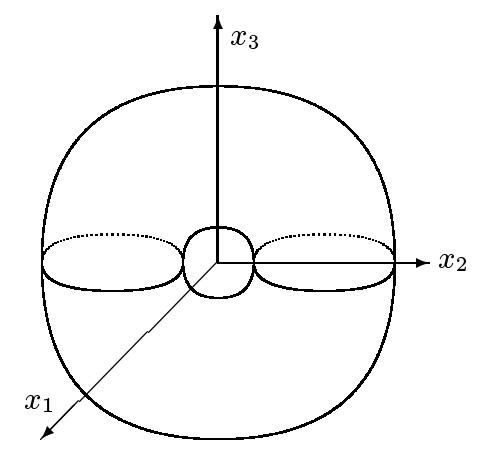

Let $A$ be the subset of $X$ with two components $A_{1}$ and $A_{2}$, where $A_{1}$ and $A_{2}$ are two solid tori in $X$ defined by

$$
A_{1}=\left\{x \in X \mid d\left(x, B_{1}\right) \leq 1 / 2\right\}, \quad A_{2}=\left\{x \in X \mid d\left(x, B_{2}\right) \leq 1 / 2\right\},
$$

where $d(\cdot, \cdot)$ is the usual metric in $\mathbb{R}^{3}$, and

$B_{1}=\left\{\left(0, x_{2}, x_{3}\right) \mid\left(x_{2}-3\right)^{2}+x_{3}^{2}=1\right\}, \quad B_{2}=\left\{\left(0, x_{2}, x_{3}\right) \mid\left(x_{2}+3\right)^{2}+x_{3}^{2}=1\right\}$.
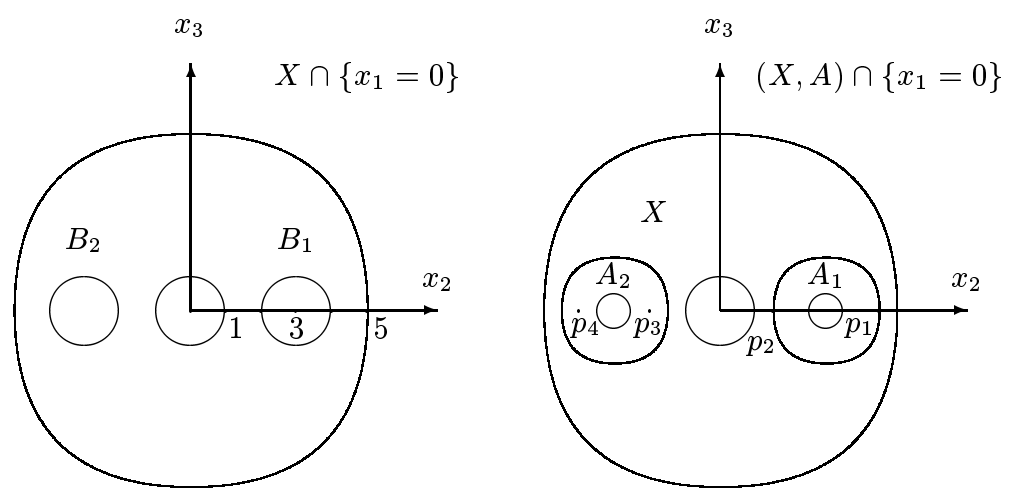

A map $f:(X, A) \rightarrow(X, A)$ is defined by $f\left(x_{1}, x_{2}, x_{3}\right)=\left(x_{1}, x_{2},-x_{3}\right)$.

From the definition $f$ has two fixed point classes $\mathbf{F}_{1}$ and $\mathbf{F}_{2}$, where

$$
\begin{aligned}
& \mathbf{F}_{1}=\left\{\left(x_{1}, x_{2}, 0\right) \mid x_{1}^{2}+\left(x_{2}-3\right)^{2} \leq 4\right\}, \\
& \mathbf{F}_{2}=\left\{\left(x_{1}, x_{2}, 0\right) \mid x_{1}^{2}+\left(x_{2}+3\right)^{2} \leq 4\right\} .
\end{aligned}
$$

We can calculate that $\operatorname{ind}\left(f, \mathbf{F}_{1}\right)=\operatorname{ind}\left(f, \mathbf{F}_{2}\right)=1$, so $N(f)=2$.

Write $f_{1}: A_{1} \rightarrow A_{1}$ and $f_{2}: A_{2} \rightarrow A_{2}$ for the restrictions of $f$ to $A_{1}$ and $A_{2}$. Then $f_{1}$ and $f_{2}$ have the same homotopy type as $f$ with Fix $f_{1} \subset \mathbf{F}_{1}$, Fix $f_{2} \subset \mathbf{F}_{2}$. Since $L(f)=L\left(f_{1}\right)=L\left(f_{2}\right)=2, \mathbf{F}_{1}$ and $\mathbf{F}_{2}$ are common and do not assume their indices in $A$. Thus, $N(f, \bar{f})=2, \widetilde{n}(f ; X, A)=2$ and $N(f ; X, A)=N(\bar{f})+N(f)-N(f, \bar{f})=4+2-2=4$.

Let $P=\left\{\left(0,4 \frac{1}{2}, 0\right),\left(0,1 \frac{1}{2}, 0\right),(0,-2,0),(0,-4,0)\right\}=\left\{p_{1}, p_{2}, p_{3}, p_{4}\right\}$. Then $|P \cap \operatorname{Int}(A)|=2=N(\bar{f})-\widetilde{n}(f ; X, A),|P \cap \operatorname{Bd}(A)|=2=\widetilde{n}(f ; X, A)$ 
and $|P \cap(X-A)|=0=N(f ; X-A)$. We shall show that $P$ cannot be the fixed point set of any map in the homotopy class of $f$.

Suppose $g:(X, A) \rightarrow(X, A)$ is homotopic to $f$ and Fix $g=P$, write $g_{1}: A_{1} \rightarrow A_{1}$ and $g_{2}: A_{2} \rightarrow A_{2}$ for the restrictions of $g$ to $A_{1}$ and $A_{2}$. Then $g_{1} \simeq f_{1}, g_{2} \simeq f_{2}$. It follows that $N\left(g_{1}\right)=N\left(g_{2}\right)=2$. Since Fix $g_{1}=\left\{p_{1}, p_{2}\right\}$ and Fix $g_{2}=\left\{p_{3}, p_{4}\right\}$, by the homotopy invariance of fixed point index we have

$$
\operatorname{ind}\left(g_{1}, p_{1}\right)=\operatorname{ind}\left(g_{1}, p_{2}\right)=\operatorname{ind}\left(g_{2}, p_{3}\right)=\operatorname{ind}\left(g_{2}, p_{4}\right)=1 \text {. }
$$

Also, $g$ has two essential fixed point classes $\mathbf{G}_{1}$ and $\mathbf{G}_{2}$, which are homotopy related to $\mathbf{F}_{1}$ and $\mathbf{F}_{2}$ respectively. Since $\mathbf{F}_{1}$ contains two essential fixed point classes of $f_{1}$ and since $\mathbf{F}_{2}$ contains two fixed point classes of $f_{2}, \mathbf{G}_{1}$ and $\mathbf{G}_{2}$ contain two essential fixed point classes of $g_{1}$ and $g_{2}$ respectively. Hence, $\mathbf{G}_{1}=\left\{p_{1}, p_{2}\right\}$ and $\mathbf{G}_{2}=\left\{p_{3}, p_{4}\right\}$. Since $\mathbf{G}_{2}$ is contained $\operatorname{in} \operatorname{Int}\left(A_{2}\right)$, we have

$$
\operatorname{ind}\left(g, \mathbf{G}_{2}\right)=\operatorname{ind}\left(g, p_{3}\right)+\operatorname{ind}\left(g, p_{4}\right)=\operatorname{ind}\left(g_{2}, p_{3}\right)+\operatorname{ind}\left(g_{2}, p_{4}\right)=2 .
$$

On the other hand, $\operatorname{ind}\left(g, \mathbf{G}_{2}\right)=\operatorname{ind}\left(f, \mathbf{F}_{2}\right)=1$. This is a contradiction. So we have proved that $P$ cannot be the fixed point set of any map in the homotopy class of $f$.

REMARK 1.5. The example above does not show that Theorem 1.3 is false. In fact, we can pick another finite set

$$
P^{\prime}=\left\{(0,4,0),\left(0,1 \frac{1}{2}, 0\right),\left(0,-1 \frac{1}{2}, 0\right),(0,-4,0)\right\} .
$$

Then $\left|P^{\prime} \cap \operatorname{Int}(A)\right|=|P \cap \operatorname{Int}(A)|=2,\left|P^{\prime} \cap(X-A)\right|=|P \cap(X-A)|=0$. It is obvious that $P^{\prime}$ can be the fixed point set of a map in the homotopy class of $f$.

It is the purpose of this paper to give a theoretical reason for this phenomenon. We shall show that there exist more restrictions imposed on minimal fixed point sets of relative maps and hence we obtain some conditions for the realization of the minimal fixed point set, i.e. give an answer to Problem 1.1.

We assume the reader is familiar with the results and technique in relative Nielsen theory which were introduced in [5], [6] and [8]. In this paper, all the fixed point classes are those in the sense of lifting classes, i.e. the empty fixed point classes are taken into account. Background material can be found in [4].

2. Bipartite graphs and matchings. In this section, we present some matching theorems. Similar conclusions can be found in [7, Sec. 4]. Here, some adjustments are made for our purpose. On the way, we introduce some notations in order to give a brief definition of relative Nielsen numbers in the next section. 
A bipartite graph $\mathcal{G}$ is a graph represented by a triple $\mathcal{G}=(\mathcal{A}, \mathcal{B}, \mathcal{E})$, where the vertex set of $\mathcal{G}$ is the disjoint union of $\mathcal{A}$ and $\mathcal{B}$, and the edge set $\mathcal{E}$ of $\mathcal{G}$ is such that each edge joins a vertex in $\mathcal{A}$ to a vertex in $\mathcal{B}$.

For a vertex subset $V$ of $\mathcal{G}$, a $V$-semimatching $\mathcal{M}$ in $\mathcal{G}$ is a subset of $\mathcal{E}$ such that no two edges of $\mathcal{M}$ share a vertex in $V$. In particular, when $V=\mathcal{A} \cup \mathcal{B}$ is the total vertex set, a $V$-semimatching $\mathcal{M}$ is said to be a matching in $\mathcal{G}$, i.e. no two elements of $\mathcal{M}$ share a vertex.

A $V$-semimatching (or matching) $\mathcal{M}$ in $\mathcal{G}$ is said to saturate a vertex set $\mathcal{S}$ in $\mathcal{G}$ if every vertex in $\mathcal{S}$ is an endpoint of an edge in $\mathcal{M}$.

For $\mathcal{S} \subseteq \mathcal{A}$ and $\mathcal{T} \subseteq \mathcal{B}$, we define

$$
\begin{aligned}
\Gamma(\mathcal{S}) & =\{b \in \mathcal{B} \mid(a, b) \in \mathcal{E} \text { for some } a \in \mathcal{S}\} \\
\gamma(\mathcal{T}) & =\{a \in \mathcal{A} \mid(a, b) \in \mathcal{E} \text { for some } b \in \mathcal{T}\}
\end{aligned}
$$

where $(a, b)$ denotes an edge between $a$ and $b$. We shall write $\Gamma_{\mathcal{G}}(\mathcal{S})$ and $\gamma_{\mathcal{G}}(\mathcal{T})$ if a specific graph $\mathcal{G}$ is emphasized.

Lemma 2.1. A bipartite $\mathcal{G}=(\mathcal{A}, \mathcal{B}, \mathcal{E})$ admits a matching which saturates $\mathcal{B}$ if and only if, for all $\mathcal{T} \subseteq \mathcal{B},|\gamma(\mathcal{T})| \geq|\mathcal{T}|$. Symmetrically, it admits a matching which saturates $\mathcal{A}$ if and only if, for all $\mathcal{S} \subseteq \mathcal{A},|\Gamma(\mathcal{S})| \geq|\mathcal{S}|$.

Proof. See [2, Theorem 1].

This result was originally stated in terms of representatives of subsets ([2, Theorem 1]), but one can translate it into the form of a matching of a bipartite graph if we regard the membership relations as edges connecting elements and subsets of a given set (cf. [7, Sec. 4]).

TheOrem 2.2. Let $\mathcal{G}=(\mathcal{A}, \mathcal{B}, \mathcal{E})$ be a bipartite graph and $\phi: \mathcal{A} \rightarrow \mathbb{Z}$ be a correspondence from $\mathcal{A}$ to the nonnegative integers. Then there is a $\mathcal{B}$-semimatching $\mathcal{M}$ which saturates $\mathcal{B}$ such that $|\{b \mid(a, b) \in \mathcal{M}\}| \leq \phi(a)$ for all $a \in \mathcal{A}$ if and only if, for all $\mathcal{T} \subseteq \mathcal{B}$,

$$
\sum_{a \in \gamma(\mathcal{T})} \phi(a) \geq|\mathcal{T}|
$$

P r o of. We construct a new bipartite graph $\mathcal{G}^{\prime}=\left(\mathcal{A}^{\prime}, \mathcal{B}^{\prime}, \mathcal{E}^{\prime}\right)$ as follows: Let $\mathcal{B}^{\prime}=\mathcal{B}$ and begin with $\mathcal{A}^{\prime}=\emptyset$ and $\mathcal{E}^{\prime}=\emptyset$. For each $a \in \mathcal{A}$, add $\phi(a)$ new vertices to $\mathcal{A}^{\prime}$ and then add edges from each of these new vertices to all the vertices $b \in \Gamma_{\mathcal{G}}(a)$.

Now, observe that the hypothesis $(*)$ is equivalent to $\left|\gamma_{\mathcal{G}^{\prime}}(\mathcal{T})\right| \geq|\mathcal{T}|$ for all $\mathcal{T} \subseteq \mathcal{B}^{\prime}$. Applying Lemma 2.1 to the graph $\mathcal{G}^{\prime}$, we get a matching $\mathcal{M}^{\prime}$ in $\mathcal{G}^{\prime}$ saturating $\mathcal{B}^{\prime}$, which corresponds to a $\mathcal{B}$-semimatching $\mathcal{M}$ in $\mathcal{G}$ which saturates $\mathcal{B}$ with $|\{b \mid(a, b) \in \mathcal{M}\}| \leq \phi(a)$ for all $a \in \mathcal{A}(\mathcal{G})$. 
TheOREM 2.3. Let $\mathcal{G}=(\mathcal{A}, \mathcal{B}, \mathcal{E})$ be a bipartite graph and $\phi: \mathcal{A} \rightarrow \mathbb{Z}$ be a correspondence from $\mathcal{A}$ to the nonnegative integers. Then there is a $\mathcal{B}$-semimatching $\mathcal{M}$ such that $|\{b \mid(a, b) \in \mathcal{M}\}|=\phi(a)$ for all $a \in \mathcal{A}$ if and only if , for all $\mathcal{S} \subseteq \mathcal{A}$,

$$
\sum_{a \in \mathcal{S}} \phi(a) \leq|\Gamma(\mathcal{S})|
$$

Proof. We construct $\mathcal{G}^{\prime}=\left(\mathcal{A}^{\prime}, \mathcal{B}^{\prime}, \mathcal{E}^{\prime}\right)$ as in the proof of Theorem 2.2. The condition $(* *)$ is equivalent to $\left|\Gamma_{\mathcal{G}^{\prime}}\left(S^{\prime}\right)\right| \geq\left|S^{\prime}\right|$ for all $\mathcal{S} \subseteq \mathcal{A}^{\prime}$. Applying Lemma 2.1 to the graph $\mathcal{G}^{\prime}$, we get a matching $\mathcal{M}^{\prime}$ in $\mathcal{G}^{\prime}$ saturating $\mathcal{A}^{\prime}$, and this matching corresponds to a $\mathcal{B}$-semimatching $\mathcal{M}$ in $\mathcal{G}$ with $|\{b \mid(a, b) \in \mathcal{M}\}|=\phi(a)$ for all $a \in \mathcal{A}(\mathcal{G})$.

3. Relative Nielsen numbers and minimal fixed point sets. Let $f:(X, A) \rightarrow(X, A)$ be a self map of a pair of compact polyhedra. For any component $A_{k}$ of $A$, we write $f_{k}$ for the restriction of $f$ to $A_{k}$. As in [8, Sec. 2] (cf. [4, Ch. 1, Sec. 1]), we can define, via lifting classes, the set $\operatorname{FPC}(f)$ of fixed point classes of $f: X \rightarrow X$ and the set $\operatorname{FPC}\left(f_{k}\right)$ of those of $f_{k}$ if $f_{k}: A_{k} \rightarrow A_{k}$ is a self map. The set $\operatorname{FPC}\left(f_{k}\right)$ of fixed point classes of $f_{k}$ is regarded as being empty if $f_{k}$ is not a self map on $A_{k}$, i.e. $f\left(A_{k}\right) \nsubseteq A_{k}$. For each $f_{k}$, there is a correspondence

$$
i_{k, \mathrm{FPC}}: \operatorname{FPC}\left(f_{k}\right) \rightarrow \mathrm{FPC}(f) .
$$

A fixed point class $\mathbf{F}_{k}$ of $f_{k}$ is contained in a fixed point class $\mathbf{F}$ of $f: X \rightarrow X$ if and only if $i_{k, \mathrm{FPC}}\left(\mathbf{F}_{k}\right)=\mathbf{F}$. Letting $\operatorname{FPC}(\bar{f})=\bigcup \operatorname{FPC}\left(f_{k}\right)$ be the disjoint union of the fixed point classes of $f$ on all components of $A$, we get a correspondence

$$
i_{\mathrm{FPC}}: \operatorname{FPC}(\bar{f}) \rightarrow \operatorname{FPC}(f) .
$$

Furthermore, we write $\mathrm{FPC}_{\mathrm{e}}(\cdot)$ and $\mathrm{FPC}_{\mathrm{i}}(\cdot)$ for the sets of essential and inessential fixed point classes respectively. With this notation, we have $N(f ; X-A)=\left|\mathrm{FPC}_{\mathrm{e}}(f)-i_{\mathrm{FPC}}(\mathrm{FPC}(\bar{f}))\right|$. Write $\mathcal{F}$ for the set of fixed point classes of $f$ which do not assume their indices in $A$, i.e.

$$
\mathcal{F}=\{\mathbf{F} \in \operatorname{FPC}(f) \mid \operatorname{ind}(f, \mathbf{F}) \neq \operatorname{ind}(\bar{f}, \mathbf{F} \cap A)\} .
$$

We define

$$
\begin{aligned}
& \mathcal{F}_{1}=\mathcal{F} \cap i_{\mathrm{FPC}}\left(\operatorname{FPC}_{\mathrm{e}}(\bar{f})\right), \\
& \mathcal{F}_{2}=\left(\mathrm{FPC}_{\mathrm{e}}(f) \cap i_{\mathrm{FPC}}\left(\mathrm{FPC}_{\mathrm{i}}(\bar{f})\right)-i_{\mathrm{FPC}}\left(\mathrm{FPC}_{\mathrm{e}}(\bar{f})\right) .\right.
\end{aligned}
$$

Then $\mathcal{F}_{1} \cup \mathcal{F}_{2} \subseteq \mathcal{F}$, and $\mathcal{F}-\left(\mathcal{F}_{1} \cup \mathcal{F}_{2}\right)$ is the set of essential and weakly noncommon fixed point classes of $f$. Hence, $\left|\mathcal{F}_{1}\right|=\widetilde{n}(f ; X, A),\left|\mathcal{F}_{2}\right|=$ $N_{1010}(f ; X, A),\left|\mathcal{F}-\left(\mathcal{F}_{1} \cup \mathcal{F}_{2}\right)\right|=N(f ; X-A)$ and $|\mathcal{F}|=\widetilde{N}(f ; X, A)$. 
Writing $\mathcal{A}$ for the set of all components of $A$, we construct two bipartite graphs as follows:

$$
\begin{aligned}
& \mathcal{G}_{1}=\left(\mathcal{A}, \mathcal{F}_{1}, \mathcal{E}_{1}=\left\{\left(A_{j}, \mathbf{F}\right) \mid \mathbf{F} \in i_{j, \mathrm{FPC}}\left(\mathrm{FPC}_{\mathrm{e}}\left(f_{j}\right)\right)\right\}\right), \\
& \mathcal{G}_{2}=\left(\mathcal{A}, \mathcal{F}_{2}, \mathcal{E}_{2}=\left\{\left(A_{j}, \mathbf{F}\right) \mid \mathbf{F} \in i_{j, \mathrm{FPC}}\left(\operatorname{FPC}\left(f_{j}\right)\right)\right\}\right) .
\end{aligned}
$$

Definition 3.1. For any $\mathcal{S} \subseteq \mathcal{A}$, we define

$$
\widetilde{n}(f ; X, \mathcal{S}):=\left|\Gamma_{\mathcal{G}_{1}}(\mathcal{S})\right|, \quad n(f ; X, \mathcal{S}):=\left|\Gamma_{\mathcal{G}_{2}}(\mathcal{S})\right| .
$$

It is obvious that for any $\mathcal{S}=\left\{A_{1}, \ldots, A_{k}\right\} \subseteq \mathcal{A}, \widetilde{n}(f ; X, \mathcal{S})$ is the number of common fixed point classes of $f$ which do not assume their indices in $A$ and contain essential fixed point classes of $f_{j}: A_{j} \rightarrow A_{j}$ for an $A_{j} \in \mathcal{S}$, and $n(f ; X, \mathcal{S})$ is the number of noncommon and essential fixed point classes of $f$ which contain (inessential) fixed point classes of $f_{j}: A_{j} \rightarrow A_{j}$ for an $A_{j} \in \mathcal{S}$. Clearly, we have

Corollary 3.2. For any $\mathcal{S} \subseteq \mathcal{A}$,

$$
\begin{gathered}
0 \leq \widetilde{n}(f ; X, A)-\widetilde{n}(f ; X, \mathcal{A}-\mathcal{S}) \leq \widetilde{n}(f ; X, \mathcal{S}) \leq \min \left(\widetilde{n}(f ; X, A), \sum_{A_{j} \in \mathcal{S}} N\left(f_{j}\right)\right), \\
0 \leq N_{1010}(f ; X, A)-n(f ; X, \mathcal{A}-\mathcal{S}) \leq n(f ; X, \mathcal{S}) \\
\quad \leq \min \left(N_{1010}(f ; X, A), \sum_{A_{j} \in \mathcal{S}}\left(R\left(f_{j}\right)-N\left(f_{j}\right)\right)\right),
\end{gathered}
$$

in particular, $\widetilde{n}(f ; X, \emptyset)=n(f ; X, \emptyset)=0, \widetilde{n}(f ; X, \mathcal{A})=\widetilde{n}(f ; X, A), n(f ; X, \mathcal{A})$ $=N_{1010}(f ; X, A)$.

So, the notation $\widetilde{n}(f ; X, \mathcal{A})$ coincides with the original $\widetilde{n}(f ; X, A)$ in $[6]$ if we regard $\mathcal{A}$ as $A$. In what follows, we shall treat $\mathcal{S} \subseteq \mathcal{A}$ as a collection of components of $A$ or the union of the elements in $\mathcal{S}$, depending on context. Thus, $\operatorname{Bd}(\mathcal{S})$ and $\operatorname{Int}(\mathcal{S})$ are thought of as the sets $\bigcup_{A_{j} \in \mathcal{S}} \operatorname{Bd}\left(A_{j}\right)$ and $\bigcup_{A_{j} \in \mathcal{S}} \operatorname{Int}\left(A_{j}\right)$ respectively.

From the proof of the lower bound property of the relative Nielsen number $N(f ; X, A)$ in [5], we get

LEMMA 3.3. If $f$ has a minimal fixed point set, then, for any fixed point class $\mathbf{F}$ of $f$,

$$
|\mathbf{F}|= \begin{cases}\left|i_{\mathrm{FPC}}^{-1}(\mathbf{F}) \cap i_{\mathrm{FPC}}\left(\mathrm{FPC}_{\mathrm{e}}(\bar{f})\right)\right| & \text { if } \mathbf{F} \text { is common, } \\ 1 & \text { if } \mathbf{F} \text { is noncommon and essential, } \\ 0 & \text { otherwise. }\end{cases}
$$

For any $\overline{\mathbf{F}} \in \mathrm{FPC}_{\mathrm{e}}(\bar{f}),|\overline{\mathbf{F}}|=1$.

Lemma 3.4. Let $\mathcal{S} \subseteq \mathcal{A}$ and $\mathbf{F} \in \mathcal{F}_{1}-\Gamma_{\mathcal{G}_{1}}(\mathcal{A}-\mathcal{S})$. If $f$ has a minimal fixed point set, then $\mathbf{F} \subseteq \mathcal{S}$ and $\mathbf{F} \cap \operatorname{Bd}(\mathcal{S}) \neq \emptyset$. 
Pr o of. As $\mathbf{F} \in \mathcal{F}_{1}, \mathbf{F}$ is common. Since $f$ has a minimal fixed point set, by Lemma 3.3, $|\mathbf{F}|$ is equal to the number of essential fixed point classes of $\bar{f}$ which are contained in $\mathbf{F}$; each of them contains exactly one fixed point of $\bar{f}$. Because $\mathbf{F} \notin \Gamma_{\mathcal{G}_{1}}(\mathcal{A}-\mathcal{S}), \mathbf{F}$ does not lie in the image of $i_{j, \mathrm{FPC}}\left(\mathrm{FPC}_{\mathrm{e}}\left(f_{j}\right)\right)$ for any $A_{j} \notin \mathcal{S}$, and therefore each essential fixed class of $\bar{f}$ contained in $\mathbf{F}$ is a fixed point class of $f_{j}: A_{j} \rightarrow A_{j}$ for some $A_{j} \in \mathcal{S}$. Thus, $\mathbf{F} \subseteq \mathcal{S}$. Moreover, $\mathbf{F}$ does not assume its index in $A$, which implies that $\mathbf{F} \cap \mathrm{Cl}(X-A) \neq \emptyset$. So, we have $\mathbf{F} \cap \operatorname{Bd}(\mathcal{S}) \neq \emptyset$.

Notice that $\left|\mathcal{F}_{1}-\Gamma_{\mathcal{G}_{1}}(\mathcal{A}-\mathcal{S})\right|=\widetilde{n}(f ; X, A)-\widetilde{n}(f ; X, \mathcal{A}-\mathcal{S})$. The second conclusion of Lemma 3.4 implies

Theorem 3.5. Let $\mathcal{S} \subseteq \mathcal{A}$. If $f$ has a minimal fixed point set, then $f$ has at least $\widetilde{n}(f ; X, A)-\widetilde{n}(f ; X, \mathcal{A}-\mathcal{S})$ fixed points on $\operatorname{Bd}(\mathcal{S})$.

TheOREM 3.6. Let $\mathcal{S} \subseteq \mathcal{A}$. If $f$ has a minimal fixed point set, then $f$ has at most $\sum_{A_{j} \in \mathcal{S}} N\left(f_{j}\right)-\widetilde{n}(f ; X, A)+\widetilde{n}(f ; X, \mathcal{A}-\mathcal{S})$ fixed points in $\operatorname{Int}(\mathcal{S})$.

Proof. Suppose

$$
\mid \text { Fix } f \cap \operatorname{Int}(\mathcal{S}) \mid>\sum_{A_{j} \in \mathcal{S}} N\left(f_{j}\right)-\widetilde{n}(f ; X, A)+\widetilde{n}(f ; X, \mathcal{A}-\mathcal{S}) .
$$

By Theorem 3.5, $\mid$ Fix $f \cap \operatorname{Bd}(\mathcal{S}) \mid \geq \widetilde{n}(f ; X, A)-\widetilde{n}(f ; X, \mathcal{A}-\mathcal{S})$, and so we have $\mid$ Fix $f \cap \mathcal{S} \mid>\sum_{A_{j} \in \mathcal{S}} N\left(f_{j}\right)$. Since $f$ has a minimal fixed point set, from Lemma 3.3, each essential fixed point class of $\bar{f}$ contains exactly one point. Among these $\sum_{A_{j} \in \mathcal{S}} N\left(f_{j}\right)$ fixed points in essential fixed point classes of $\bar{f}$, by Theorem 3.5 , there are $\widetilde{n}(f ; X, A)-\widetilde{n}(f ; X, \mathcal{A}-\mathcal{S})$ points that lie on $\operatorname{Bd}(\mathcal{S})$. Thus, there must be a fixed point $x \in \operatorname{Int}(\mathcal{S})$ which belongs to an inessential fixed point class $\mathbf{F}_{j}$ of $f: A_{j} \rightarrow A_{j}$ where $x \in A_{j} \in \mathcal{S}$. Let $\mathbf{F}=i_{j, \mathrm{FPC}}\left(\mathbf{F}_{j}\right)$. We claim that there is a contradiction in each case of Lemma 3.3 for the fixed point class $\mathbf{F}$ of $f$.

Case (i): $\mathbf{F}$ is common. As $\mathbf{F}_{j}$ is inessential, $x$ would be different from those $\left|i_{\mathrm{FPC}}^{-1}(\mathbf{F}) \cap i_{\mathrm{FPC}}\left(\mathrm{FPC}_{\mathrm{e}}(\bar{f})\right)\right|$ fixed points.

Case (ii): $\mathbf{F}$ is noncommon and essential. Then $\mathbf{F}$ does not assume its index in $A$, we have $\mathbf{F} \cap \mathrm{Cl}(X-A) \neq \emptyset$. But $x \notin \mathrm{Cl}(X-A)$, hence $|\mathbf{F}| \geq 2 \neq 1$.

Case (iii): Trivial.

The theorem above is not a direct corollary of Theorem 3.5. In fact, a component $A_{j}$ of $A$ may contain more than $N\left(f_{j}\right)$ fixed points on $A_{j}$ even if $f$ has a minimal fixed point set. An upper bound is given in:

Theorem 3.7. Let $\mathcal{S} \subseteq \mathcal{A}$. If $f$ has a minimal fixed point set, then $f$ has at most $\sum_{A_{j} \in \mathcal{S}} N\left(f_{j}\right)+n(f ; X, \mathcal{S})$ fixed points in $\mathcal{S}$.

Pr o of. Since $f$ has a minimal fixed point set, each essential fixed point class of $\bar{f}$ contains exactly one fixed point. If $x$ is a fixed point in $\mathcal{S}$ which 
is different from these $\sum_{A_{j} \in \mathcal{S}} N\left(f_{j}\right)$ points, then $x$ belongs to an inessential fixed point class $\mathbf{F}_{j}$ of $f_{j}: A_{j} \rightarrow A_{j}$ with $A_{j} \in \mathcal{S}$. Let $\mathbf{F}=i_{j, \mathrm{FPC}}\left(\mathbf{F}_{j}\right)$. From Lemma 3.3, we see that $\mathbf{F}$ is noncommon (in case (ii)). Thus, we have $\mathbf{F} \in \mathcal{F}_{2}$, and therefore $\mathbf{F} \subseteq \Gamma_{\mathcal{G}_{2}}(\mathcal{S})$.

Note that $\left|\Gamma_{\mathcal{G}_{2}}(\mathcal{S})\right|=n(f ; X, \mathcal{S})$ and that, from Lemma 3.3, any fixed point class of $f$ in $\mathcal{F}_{2}$ contains exactly one fixed point if $f$ has a minimal fixed point set. So the theorem holds.

We summarize the lower and upper bound theorems as follows:

Theorem 3.8. Let $\mathcal{S}=\left\{A_{1}, \ldots, A_{k}\right\} \subseteq \mathcal{A}$ be $k$ components of $A$. If $f$ has a minimal fixed point set, i.e. $\mid$ Fix $f \mid=N(f ; X, A)$, then

(1) $0 \leq|\operatorname{Fix} f \cap \operatorname{Int}(\mathcal{S})| \leq \sum_{j=1}^{k} N\left(f_{j}\right)-\widetilde{n}(f ; X, A)+\widetilde{n}(f ; X, \mathcal{A}-\mathcal{S})$,

(2) $\widetilde{n}(f ; X, A)-\widetilde{n}(f ; X, \mathcal{A}-\mathcal{S}) \leq|\operatorname{Fix} f \cap \operatorname{Bd}(\mathcal{S})| \leq \sum_{j=1}^{k} N\left(f_{j}\right)+$ $n(f ; X, \mathcal{S})$,

(3) $\sum_{j=1}^{k} N\left(f_{j}\right) \leq \mid$ Fix $f \cap \mathcal{S} \mid \leq \sum_{j=1}^{k} N\left(f_{j}\right)+n(f ; X, \mathcal{S})$.

Similar to [8], we have

Theorem 3.9 (Homotopy invariance). Let $f \simeq g:(X, A) \rightarrow(X, A)$ be two homotopic maps. Then, for any $\mathcal{S} \subseteq \mathcal{A}$, we have

$$
\widetilde{n}(f ; X, \mathcal{S})=\widetilde{n}(g ; X, \mathcal{S}), \quad n(f ; X, \mathcal{S})=n(g ; X, \mathcal{S}) .
$$

REMARK 3.10. It is known that the fixed point class sets $\operatorname{FPC}(f)$ and $\operatorname{FPC}(\bar{f})$ are invariant under homotopy. So is the inclusion relation $i_{\mathrm{FPC}}$. Hence, the bipartite graphs $\mathcal{G}_{1}$ and $\mathcal{G}_{2}$ are constant for maps in a given (relative) homotopy class.

4. Realization of a minimal fixed point set. In this section, we shall show that under suitable assumptions on $(X, A)$ the conditions in Theorem 3.8 are also sufficient for the realization of a finite set $P$ with $|P|=$ $N(f ; X, A)$ as the fixed point set for a map in the homotopy class of the given map $f:(X, A) \rightarrow(X, A)$. Basic technique here is the same as in the proof of [5, Theorem 6.2] (cf. [3]).

THEOREM 4.1. Let $(X, A)$ be a pair of compact polyhedra such that

(1) $X$ is connected,

(2) $X-A$ has no local cut point and is not a 2-manifold,

(3) each component of $A$ is a Nielsen space,

(4) $A$ can be by-passed in $X$.

Let $f:(X, A) \rightarrow(X, A)$ and $P \subset X$ be a finite set with $|P|=N(f ; X, A)$. 
If , for any components $\mathcal{S}=\left\{A_{1}, \ldots, A_{k}\right\} \subseteq \mathcal{A}$,

$$
\begin{gathered}
|P \cap \operatorname{Int}(\mathcal{S})| \leq \sum_{j=1}^{k} N\left(f_{j}\right)-\widetilde{n}(f ; X, A)+\widetilde{n}(f ; X, \mathcal{A}-\mathcal{S}), \\
\sum_{j=1}^{k} N\left(f_{j}\right) \leq|P \cap \mathcal{S}| \leq \sum_{j=1}^{k} N\left(f_{j}\right)+n(f ; X, \mathcal{S}),
\end{gathered}
$$

then there is a map $g:(X, A) \rightarrow(X, A)$ homotopic to $f$ such that Fix $g=P$.

Pro of. Consider the two bigraphs $\mathcal{G}_{1}$ and $\mathcal{G}_{2}$. Define two correspondences $\phi_{1}, \phi_{2}: \mathcal{A} \rightarrow \mathbb{Z}$ by

$$
\phi_{1}\left(A_{j}\right)=N\left(f_{j}\right)-\left|\operatorname{Int}\left(A_{j}\right) \cap P\right|, \quad \phi_{2}\left(A_{j}\right)=\left|P \cap A_{j}\right|-N\left(f_{j}\right) .
$$

STEP 1. For any $\mathcal{T} \subseteq \mathcal{F}_{1}$, we have

$$
\begin{aligned}
\sum_{A_{j} \in \gamma_{\mathcal{G}_{1}}(\mathcal{T})} \phi_{1}\left(A_{j}\right) & =\sum_{A_{j} \in \gamma_{\mathcal{G}_{1}}(\mathcal{T})} N\left(f_{j}\right)-\left|\operatorname{Int}\left(\gamma_{\mathcal{G}_{1}}(\mathcal{T})\right) \cap P\right| \\
& \geq \widetilde{n}(f ; X, A)-\widetilde{n}\left(f ; X, \mathcal{A}-\gamma_{\mathcal{G}_{1}}(\mathcal{T})\right) \\
& =\left|\mathcal{F}_{1}\right|-\left|\Gamma_{\mathcal{G}_{1}}\left(\mathcal{A}-\gamma_{\mathcal{G}_{1}}(\mathcal{T})\right)\right| \\
& \geq|\mathcal{T}| .
\end{aligned}
$$

By Theorem 2.2, there is an $\mathcal{F}_{1}$-semimatching $\mathcal{M}_{1}$ in $\mathcal{G}_{1}$ such that $\left|\left\{\mathbf{F} \mid\left(A_{j}, \mathbf{F}\right) \in \mathcal{M}_{1}\right\}\right| \leq \phi_{1}\left(A_{j}\right)$ for all $A_{j} \in \mathcal{A}$.

For any $\mathcal{S} \subseteq \mathcal{A}$, we have

$$
\sum_{A_{j} \in \mathcal{S}} \phi_{2}\left(A_{j}\right)=\sum_{A_{j} \in \mathcal{S}}\left(\left|P \cap A_{j}\right|-N\left(f_{j}\right)\right) \leq n(f ; X, \mathcal{S})=\left|\Gamma_{\mathcal{G}_{2}}(\mathcal{S})\right| .
$$

By Theorem 2.3, there is an $\mathcal{F}_{2}$-semimatching $\mathcal{M}_{2}$ in $\mathcal{G}_{2}$ with $\mid\left\{\mathbf{F} \mid\left(A_{j}, \mathbf{F}\right) \in\right.$ $\left.\mathcal{M}_{2}\right\} \mid=\phi_{2}\left(A_{j}\right)$ for all $A_{j} \in \mathcal{A}$.

STEP 2. For any component $A_{j}$ of $A$, notice that

$$
\begin{aligned}
\left|P \cap \operatorname{Bd}\left(A_{j}\right)\right| & =\left|P \cap A_{j}\right|-\left|P \cap \operatorname{Int}\left(A_{j}\right)\right| \\
& =\left(\phi_{2}\left(A_{j}\right)+N\left(f_{j}\right)\right)-\left(N\left(f_{j}\right)-\phi_{1}\left(A_{j}\right)\right) \\
& =\phi_{1}\left(A_{j}\right)+\phi_{2}\left(A_{j}\right) \\
& \geq\left|\left\{\mathbf{F} \mid\left(A_{j}, \mathbf{F}\right) \in \mathcal{M}_{1}\right\}\right|+\left|\left\{\mathbf{F} \mid\left(A_{j}, \mathbf{F}\right) \in \mathcal{M}_{2}\right\}\right| .
\end{aligned}
$$

Thus, we can label $\left|\left\{\mathbf{F} \mid\left(A_{j}, \mathbf{F}\right) \in \mathcal{M}_{1}\right\}\right|+\left|\left\{\mathbf{F} \mid\left(A_{j}, \mathbf{F}\right) \in \mathcal{M}_{2}\right\}\right|$ points in $P \cap \operatorname{Bd}\left(A_{j}\right)$ with fixed point classes of $f$ which are the vertices of the edge sets $\mathcal{M}_{1}$ or $\mathcal{M}_{2}$.

Altogether, we thus get $\left|\mathcal{M}_{1}\right|+\left|\mathcal{M}_{2}\right|$ labelled points in $P \cap \operatorname{Bd}(A)$. Since $\mathcal{M}_{1}$ is an $\mathcal{F}_{1}$-semimatching in $\mathcal{G}_{1}$ saturating $\mathcal{F}_{1}$, for each $\mathbf{F} \in \mathcal{F}_{1}$ there exists exactly one point labelled by $\mathbf{F}$. The $\mathcal{F}_{2}$-semimatching $\mathcal{M}_{2}$ implies that for 
any $\mathbf{F} \in \mathcal{F}_{2}$ there is at most one point which is labelled by $\mathbf{F}$. (Some fixed point classes in $\mathcal{F}_{2}$ have not been labelled the fixed points in these will be left in the complement $X-A$ later).

STEP 3. For each component $A_{k}$ of $A$, we can homotope $f_{k}: A_{k} \rightarrow A_{k}$ to a map $f_{k}^{(1)}: A_{k} \rightarrow A_{k}$ which has $N\left(f_{k}\right)$ fixed points lying in $A_{k}-P$.

Since $\mathcal{M}_{1}$ saturates $\mathcal{F}_{1}$, for each $\mathbf{F} \in \mathcal{F}_{1}$ there is a unique edge $\left(A_{j}, \mathbf{F}\right)$ in $\mathcal{M}_{1}$. Let $p \in P \cap \operatorname{Bd}\left(A_{j}\right)$ be the point labelled by $\mathbf{F}$. From the definition of the bipartite graph $\mathcal{G}_{1}, \mathbf{F}$ contains an essential fixed point class $\mathbf{F}_{j}$ of $f_{j}$. We move the single fixed point $x$ in $\mathbf{F}_{j}$ to the point $p$.

Repeat the procedure above for every element in $\mathcal{M}_{1}$. We shall move the $\left|\mathcal{F}_{1}\right|$ fixed points to the labelled points of fixed point classes in $\mathcal{F}_{1}$. Moving the other fixed points to those $N(\bar{f})-\left|\mathcal{F}_{1}\right|$ points in $P \cap A$ which are not labelled, we get a map $\bar{f}^{(2)}: A \rightarrow A$ homotopic to $\bar{f}: A \rightarrow A$ with the properties:

(1) $\left.\bar{f}^{(2)}\right|_{A_{j}}: A_{j} \rightarrow A_{j}$ has $N\left(f_{j}\right)$ fixed points for all components $A_{j}$ of $A$,

(2) $P \cap \operatorname{Int}(A) \subseteq \operatorname{Fix} \bar{f}^{(2)} \subseteq P \cap A$,

(3) for each edge $\left(A_{j}, \mathbf{F}\right) \in \mathcal{M}_{1}$, there is a fixed point $p \in \operatorname{Bd}\left(A_{j}\right) \cap \mathbf{F}$ with $p \in \mathbf{F}_{j} \in \operatorname{FPC}_{\mathrm{e}}\left(\bar{f}^{(2)}\right)$.

SteP 4. Consider an edge $\left(A_{j}, \mathbf{F}\right) \in \mathcal{M}_{2}$. From the definition of the bipartite graph $\mathcal{G}_{2}, \mathbf{F}$ contains an inessential fixed point class $\mathbf{F}_{j}$ of $f_{j}$ : $A_{j} \rightarrow A_{j}$ for this $A_{j}$. Write $p$ for the point in $A_{j} \cap P$ which is labelled by $\mathbf{F}$. As in the proof of $[8$, Theorem 3.6], we can create a fixed point at $p$ which is contained in $\mathbf{F}_{j}$, and therefore in $\mathbf{F}$. Thus we get a map $\bar{f}^{(3)}: A \rightarrow A$ homotopic to $\bar{f}: A \rightarrow A$ such that

(1) $\operatorname{Fix} \bar{f}^{(3)}=P \cap A$,

(2) for each edge $\left(A_{j}, \mathbf{F}\right) \in \mathcal{M}_{1}$, there is a fixed point $p \in \operatorname{Bd}\left(A_{j}\right) \cap \mathbf{F}$ with $p \in \mathbf{F}_{j} \in \operatorname{FPC}_{\mathrm{e}}\left(\bar{f}^{(3)}\right)$,

(3) for each edge $\left(A_{j}, \mathbf{F}\right) \in \mathcal{M}_{2}$, there is a fixed point $p \in \operatorname{Bd}\left(A_{j}\right) \cap \mathbf{F}$ with $p \in \mathbf{F}_{j} \in \mathrm{FPC}_{\mathrm{i}}\left(\bar{f}^{(3)}\right)$.

STEP 5. Using the homotopy extension property, we can extend $\bar{f}^{(3)}$ : $A \rightarrow A$ to a map $f^{(4)}:(X, A) \rightarrow(X, A)$ which is relatively homotopic to $f:(X, A) \rightarrow(X, A)$. From [5, Theorem 4.1], we can assume that the number of fixed points of $f^{(4)}$ in $X-A$ is finite, each fixed point of $f^{(4)}$ in $X-A$ lies in a maximal simplex, and there is a neighborhood $N(A)$ of $A$ in $X$ such that $f^{(4)}(N(A)) \subseteq A$.

Since $A$ can be by-passed in $X$ and since $X-A$ has no local cut point, we can unite the fixed points in $X-A$ which are in the same fixed point class to one point. Deleting the fixed points in $X-A$ of zero indices, we get a map $f^{(5)}:(X, A) \rightarrow(X, A)$ homotopic to $f$ such that each fixed point class of $f^{(5)}$ contains at most one fixed point in $X-A$. 
The existence of the neighborhood $N(A)$ of $A$ ensures that each fixed point $x$ of $f^{(5)}$ in $A$ has the same index as a fixed point of the restriction $\bar{f}^{(5)}$ of $f^{(5)}$ to $A$, i.e. $\operatorname{ind}\left(f^{(5)}, x\right)=\operatorname{ind}\left(\bar{f}^{(5)}, x\right)$, and therefore a fixed point class of $f^{(5)}$ contains one point in $X-A$ if and only if it does not assume its index in $A$. Thus, we have:

(1) $\operatorname{Fix} \bar{f}^{(5)}=P \cap A$,

(2) for each edge $\left(A_{j}, \mathbf{F}\right) \in \mathcal{M}_{1}$, there is a fixed point $p \in \operatorname{Bd}\left(A_{j}\right) \cap \mathbf{F}$ with $p \in \mathbf{F}_{j} \in \operatorname{FPC}_{\mathrm{e}}\left(\bar{f}^{(5)}\right)$,

(3) for each edge $\left(A_{j}, \mathbf{F}\right) \in \mathcal{M}_{2}$, there is a fixed point $p \in \operatorname{Bd}\left(A_{j}\right) \cap \mathbf{F}$ with $p \in \mathbf{F}_{j} \in \operatorname{FPC}_{\mathrm{i}}\left(\bar{f}^{(5)}\right)$,

(4) $\left|(X-A) \cap \operatorname{Fix} f^{(5)}\right|=\widetilde{N}(f ; X, A)=\left|\mathcal{F}_{1}\right|+\left|\mathcal{F}_{2}\right|+N(f ; X-A)$,

(5) $\left|\operatorname{Fix} f^{(5)}\right|=N(\bar{f})+\left|\mathcal{M}_{2}\right|+\left|\mathcal{F}_{1}\right|+\left|\mathcal{F}_{2}\right|+N(f ; X-A)$.

STEP 6. For any fixed point class $\mathbf{F}$ of $f^{(5)}$ which is a vertex of the matching $\mathcal{M}_{1}$ or $\mathcal{M}_{2}$, by Step 2 , there is a point $a \in \mathbf{F} \cap \operatorname{Bd}(A)$. Hence, we can map the unique point in $\mathbf{F} \cap(X-A)$ to $a$. After doing this for all edges of $\mathcal{M}_{1}$ and $\mathcal{M}_{2}$, we get a map $f^{(6)}:(X, A) \rightarrow(X, A)$ homotopic to $f^{(5)}$ such that $\left.f^{(6)}\right|_{A}=\left.f^{(5)}\right|_{A}$ and

$$
\begin{aligned}
\left|\operatorname{Fix} f^{(6)}\right| & =\left|\operatorname{Fix} f^{(5)}\right|-\left(\left|\mathcal{M}_{1}\right|+\left|\mathcal{M}_{2}\right|\right)=\left|\operatorname{Fix} f^{(5)}\right|-\left(\left|\mathcal{F}_{1}\right|+\left|\mathcal{M}_{2}\right|\right) \\
& =N(\bar{f})+\left|\mathcal{F}_{2}\right|+N(f ; X-A) \\
& =N(\bar{f})+N_{1010}(f ; X, A)+N(f ; X-A)=N(f ; X, A) .
\end{aligned}
$$

Notice that $|P \cap(X-A)|=|P|-|P \cap A|=\mid$ Fix $f^{(6)}|-| A \cap \operatorname{Fix} f^{(6)} \mid$. Moving these fixed points on $X-A$ to $P \cap(X-A)$, we get the desired map $g:(X, A) \rightarrow(X, A)$.

Combining the theorem above with Theorem 3.8, we get

TheOREM 4.2. Let $(X, A)$ be a pair of compact polyhedra such that:

(1) $X$ is connected,

(2) $X-A$ has no local cut point and is not a 2-manifold,

(3) each component of $A$ is a Nielsen space,

(4) $A$ can be by-passed in $X$.

Let $f:(X, A) \rightarrow(X, A)$. A finite set $P \subset X$ with $|P|=N(f ; X, A)$ is a fixed point set of a map in the (relative) homotopy class of $f$ if and only if, for any components $\mathcal{S}=\left\{A_{1}, \ldots, A_{k}\right\} \subseteq \mathcal{A}$,

$$
\begin{gathered}
|P \cap \operatorname{Int}(\mathcal{S})| \leq \sum_{j=1}^{k} N\left(f_{j}\right)-\widetilde{n}(f ; X, A)+\widetilde{n}(f ; X, \mathcal{A}-\mathcal{S}), \\
\sum_{j=1}^{k} N\left(f_{j}\right) \leq|P \cap \mathcal{S}| \leq \sum_{j=1}^{k} N\left(f_{j}\right)+n(f ; X, \mathcal{S}) .
\end{gathered}
$$


We know that $\sum_{j=1}^{k} N\left(f_{j}\right)-\widetilde{n}(f ; X, A)+\widetilde{n}(f ; X, \mathcal{A}-\mathcal{S}) \geq 0$ for all $\mathcal{S} \subseteq \mathcal{A}$ from Corollary 3.2. So, the condition $|P \cap \operatorname{Int}(\mathcal{S})| \leq \sum_{j=1}^{k} N\left(f_{j}\right)-$ $\widetilde{n}(f ; X, A)+\widetilde{n}(f ; X, \mathcal{A}-\mathcal{S})$ is automatically satisfied if $\operatorname{Int}(\mathcal{S})=\emptyset$. Thus, we have

TheOREm 4.3. Let $(X, A)$ be a pair of compact polyhedra such that:

(1) $X$ is connected,

(2) $X-A$ has no local cut point and is not a 2-manifold,

(3) each component of $A$ is a Nielsen space with empty interior,

(4) $A$ can be by-passed in $X$.

Let $f:(X, A) \rightarrow(X, A)$. A finite set $P \subset X$ with $|P|=N(f ; X, A)$ is a fixed point set of a map in the (relative) homotopy class of $f$ if and only if, for any components $\mathcal{S}=\left\{A_{1}, \ldots, A_{k}\right\} \subseteq \mathcal{A}$,

$$
\sum_{j=1}^{k} N\left(f_{j}\right) \leq|P \cap \mathcal{S}| \leq \sum_{j=1}^{k} N\left(f_{j}\right)+n(f ; X, \mathcal{S}) .
$$

Finally, we go back to the example in the first section, where $(X, A)$ satisfies the assumptions in Theorem 4.2. Note that

$$
\begin{gathered}
\operatorname{FPC}(f)=\mathrm{FPC}_{\mathrm{e}}(f)=\left\{\mathbf{F}_{1}, \mathbf{F}_{2}\right\}, \\
\mathcal{F}=\left\{\mathbf{F}_{1}, \mathbf{F}_{2}\right\}, \quad \mathcal{F}_{1}=\left\{\mathbf{F}_{1}, \mathbf{F}_{2}\right\}, \quad \mathcal{F}_{2}=\emptyset .
\end{gathered}
$$

So, we have $\widetilde{N}(f ; X, A)=\widetilde{n}(f ; X, A)=2, N_{1010}(f ; X, A)=N(f ; X-A)=0$. Moreover,

$$
\begin{aligned}
& \mathcal{G}_{1}=\left(\mathcal{A}, \mathcal{F}_{1}, \mathcal{E}_{1}\right)=\left(\left\{A_{1}, A_{2}\right\},\left\{\mathbf{F}_{1}, \mathbf{F}_{2}\right\},\left\{\left(A_{1}, \mathbf{F}_{1}\right),\left(A_{2}, \mathbf{F}_{2}\right)\right\}\right), \\
& \mathcal{G}_{2}=\left(\mathcal{A}, \mathcal{F}_{2}, \mathcal{E}_{2}\right)=\left(\left\{A_{1}, A_{2}\right\}, \emptyset, \emptyset\right) .
\end{aligned}
$$

Thus, we find that $\widetilde{n}\left(f ; X,\left\{A_{1}\right\}\right)=\widetilde{n}\left(f ; X,\left\{A_{2}\right\}\right)=1$ and $n\left(f ; X,\left\{A_{1}\right\}\right)=$ $n\left(f ; X,\left\{A_{2}\right\}\right)=0$.

From Theorem 4.2, a finite set $D$ with $|D|=N(f ; X, A)=4$ can be realized as a minimal fixed point set of a map homotopic to $f$ if and only if:

$$
\begin{array}{ll}
\left|D \cap \operatorname{Int}\left(A_{1}\right)\right| \leq 2-2+1=1, & 2 \leq\left|D \cap A_{1}\right| \leq 2+0, \\
\left|D \cap \operatorname{Int}\left(A_{2}\right)\right| \leq 2-2+1=1, & 2 \leq\left|D \cap A_{2}\right| \leq 2+0, \\
\left|D \cap\left(\operatorname{Int}\left(A_{1}\right) \cup \operatorname{Int}\left(A_{2}\right)\right)\right| \leq 4-2+0=2, & 2 \leq\left|D \cap\left(A_{1} \cup A_{2}\right)\right| \leq 4+0,
\end{array}
$$

i.e.

(†) $\left|D \cap \operatorname{Int}\left(A_{1}\right)\right| \leq 1, \quad\left|D \cap \operatorname{Int}\left(A_{2}\right)\right| \leq 1, \quad\left|D \cap A_{1}\right|=\left|D \cap A_{2}\right|=2$.

It is easy to see that $(\dagger)$ holds for the $P^{\prime}$ of Remark 1.5, but it does not hold for the $P$ of Example 1.4.

5. An application. In this section, we shall make use of the relative Nielsen numbers defined in Section 3 to give a new lower bound for the 
number of fixed points of a map $f:(X, A) \rightarrow(X, A)$ on $\mathrm{Cl}(X-A)$, which is larger than the lower bound $\widetilde{N}(f ; X, A)$ in [6].

First, we make a convention: write $\mathcal{S}_{0}=\left\{A_{j} \in \mathcal{A} \mid \operatorname{Int}\left(A_{j}\right)=\emptyset\right\}$ for the set of all components of $A$ with empty interiors. For a relative map $f:(X, A) \rightarrow(X, A)$, we make

DEFINITION 5.1.

$$
\widetilde{N}^{\prime}(f ; X, A):=\widetilde{N}(f ; X, A)+\sum_{A_{j} \in \mathcal{S}_{0}} N\left(f_{j}\right)-\widetilde{n}\left(f ; X, \mathcal{S}_{0}\right) .
$$

It is clear that $\widetilde{N}^{\prime}(f ; X, A)$ is a relative homotopy invariant. Moreover, we have

Proposition 5.2.

$$
\widetilde{N}(f ; X, A) \leq \tilde{N}^{\prime}(f ; X, A) \leq N(f ; X, A) .
$$

P r o of. We have $\widetilde{n}(f ; X, A)-\widetilde{n}\left(f ; X, \mathcal{S}_{0}\right) \leq \sum_{A_{j} \in \mathcal{A}-\mathcal{S}_{0}} N\left(f_{j}\right)$ by Corollary 3.2. Thus,

$$
\begin{aligned}
\widetilde{N}^{\prime}(f ; X, A)= & \widetilde{N}(f ; X, A)+\sum_{A_{j} \in \mathcal{S}_{0}} N\left(f_{j}\right)-\widetilde{n}\left(f ; X, \mathcal{S}_{0}\right) \\
= & N(f ; X-A)+N_{1010}(f ; X, A)+\widetilde{n}(f ; X, A) \\
& +\sum_{A_{j} \in \mathcal{S}_{0}} N\left(f_{j}\right)-\widetilde{n}\left(f ; X, \mathcal{S}_{0}\right) \\
\leq & N(f ; X-A)+N_{1010}(f ; X, A)+\sum_{A_{j} \in \mathcal{S}_{0}} N\left(f_{j}\right) \\
& +\sum_{A_{j} \in \mathcal{A}-\mathcal{S}_{0}} N\left(f_{j}\right) \\
= & N(f ; X-A)+N_{1010}(f ; X, A)+N(\bar{f})=N(f ; X, A)
\end{aligned}
$$

(cf. [9, Theorem 3.9]). The left inequality is a direct consequence of Corollary 3.2 .

From Corollary 3.2, we also have

Proposition 5.3. If $\operatorname{Int}(A)=\emptyset$, i.e. $\mathcal{S}_{0}=\mathcal{A}$, then $\widetilde{N}^{\prime}(f ; X, A)=$ $N(f ; X, A)$. If every component of $A$ has a nonempty interior, i.e. $\mathcal{S}_{0}=\emptyset$, then $\widetilde{N}^{\prime}(f ; X, A)=\widetilde{N}(f ; X, A)$.

The next example will show that $\tilde{N}^{\prime}(f ; X, A)$ is different from both $\widetilde{N}(f ; X, A)$ and $N(f ; X, A)$ in general.

EXAmple 5.4. Let $X=\left\{(x, y) \in \mathbb{R}^{2} \mid 1 \leq x^{2}+y^{2} \leq 9\right\}$ be an annulus in $\mathbb{R}^{2}$, and let the subspace $A$ consist of two components $A_{1}$ and $A_{2}$, where 
$A_{1}=\{(0,2)\}$ is a single point and $A_{2}=\left\{(x, y) \in \mathbb{R}^{2} \mid x^{2}+(y+2)^{2} \leq 1\right\}$ is a disc in $X$. The map $f:(X, A) \rightarrow(X, A)$ is a reflection defined by $f(x, y)=(-x, y)$.

We see that $f$ has two fixed point classes, $\mathbf{F}_{1}=\{(0, y) \in X \mid 1 \leq y \leq 3\}$ and $\mathbf{F}_{2}=\{(0, y) \in X \mid-3 \leq y \leq-1\}$. Both have the same index 1 , and hence assume their indices in $A$. So, $\widetilde{N}(f ; X, A)=\widetilde{n}(f ; X, A)=0$. Since $\mathcal{S}_{0}=\left\{A_{1}\right\}$, we have $\widetilde{N}^{\prime}(f ; X, A)=\widetilde{N}(f ; X, A)+\sum_{A_{j} \in \mathcal{S}_{0}} N\left(f_{j}\right)-$ $\widetilde{n}\left(f ; X, \mathcal{S}_{0}\right)=0+1-0=1$. Since $\mathbf{F}_{1}$ contains the unique fixed point class of $f_{1}: A_{1} \rightarrow A_{1}, \mathbf{F}_{1}$ is common. So is $\mathbf{F}_{2}$. Thus, $N(f ; X, A)=N(f)+N(\bar{f})-$ $N(f, \bar{f})=2+2-2=2$. So, in this example, $\widetilde{N}(f ; X, A)<\widetilde{N}^{\prime}(f ; X, A)<$ $N(f ; X, A)$.

THEOREM 5.5 (Lower bound theorem). Each map in the relative homotopy class of $f:(X, A) \rightarrow(X, A)$ has at least $\widetilde{N}^{\prime}(f ; X, A)$ fixed points on $\mathrm{Cl}(X-A)$.

P r o of. Since $N^{\prime}(f ; X, A)$ is a homotopy invariant, it is sufficient to show that $N^{\prime}(f ; X, A)$ is a lower bound for the number of fixed points of $f$ on $\mathrm{Cl}(X-A)$.

Notice that $\widetilde{N}(f ; X, A)=N(f ; X-A)+N_{1010}(f ; X, A)+\widetilde{n}(f ; X, A)$. Thus

$$
\begin{aligned}
\widetilde{N}^{\prime}(f ; X, A)= & N(f ; X-A)+N_{1010}(f ; X, A)+\widetilde{n}(f ; X, A)-\widetilde{n}\left(f ; X, \mathcal{S}_{0}\right) \\
& +\sum_{A_{j} \in \mathcal{S}_{0}} N\left(f_{j}\right) .
\end{aligned}
$$

Observe that $N(f ; X-A)+N_{1010}(f ; X, A)+\widetilde{n}(f ; X, A)-\widetilde{n}\left(f ; X, \mathcal{S}_{0}\right)$ is just the number of fixed point classes that do not assume their indices in $A$ and do not contain any essential fixed point classes of $f_{j}: A_{j} \rightarrow A_{j}$ for an $A_{j} \in \mathcal{S}_{0}$, i.e. $\operatorname{Int}\left(A_{j}\right)=\emptyset$. Thus, each of these fixed point classes has a fixed point on $\mathrm{Cl}(X-A)$ which is different from the fixed points contained in those $\sum_{A_{j} \in S_{0}} N\left(f_{j}\right)$ essential fixed point classes of $\bar{f}$. Since $\mathcal{S}_{0} \subseteq \mathrm{Cl}(X-A)$, we get our conclusion.

Similar to [9, Theorem 4.2], from Theorem 3.8 we have

Theorem 5.6. If $f:(X, A) \rightarrow(X, A)$ has $N(f ; X, A)$ fixed points, then f has:

(1) at most $\sum_{A_{j} \in \mathcal{A}-\mathcal{S}_{0}} N\left(f_{j}\right)-\widetilde{n}(f ; X, A)+\widetilde{n}\left(f ; X, \mathcal{S}_{0}\right)$ fixed points in $\operatorname{Int}(A)$,

(2) at least $\sum_{A_{j} \in \mathcal{S}_{0}} N\left(f_{j}\right)+\widetilde{n}(f ; X, A)-\widetilde{n}\left(f ; X, \mathcal{S}_{0}\right)$ and at most $N(\bar{f})+$ $N_{1010}(f ; X, A)$ fixed points in $\operatorname{Bd}(A)$,

(3) at least $N(\bar{f})$ and at most $N(\bar{f})+N_{1010}(f ; X, A)$ fixed points in $A$, 
(4) at least $N(f ; X-A)$ and at most $N_{1010}(f ; X, A)+N(f ; X-A)$ fixed points in $X-A$,

(5) at least $\widetilde{N}^{\prime}(f ; X, A)$ and at most $N(f ; X, A)$ fixed points in $\mathrm{Cl}(X-A)$.

Comparing the conditions of the minimum theorem [6, Theorem 5.2] for $\widetilde{N}(f ; X, A)$ with the conditions of the minimum theorem [5, Theorem 6.2] for $N(f ; X, A)$, one finds that there is an extra condition: every component of $A$ has a nonempty interior, i.e. $\mathcal{S}_{0}=\emptyset$. From Proposition 5.3 , this implies that $\widetilde{N}^{\prime}(f ; X, A)=\widetilde{N}(f ; X, A)$. But the new lower bound $\widetilde{N}^{\prime}(f ; X, A)$ can be realized under the same assumptions as in [5, Theorem 6.2].

Theorem 5.7. Let $(X, A)$ be a pair of compact polyhedra such that:

(1) $X$ is connected,

(2) $X-A$ has no local cut point and is not a 2-manifold,

(3) every component of $A$ is a Nielsen space,

(4) $A$ can be by-passed in $X$.

Let $f:(X, A) \rightarrow(X, A)$. If

$$
\widetilde{n}(f ; X, A)-\widetilde{n}\left(f ; X, \mathcal{S}_{0}\right) \leq k_{1} \leq \sum_{A_{j} \in \mathcal{A}-\mathcal{S}_{0}} N\left(f_{j}\right)
$$

and $0 \leq k_{2} \leq N_{1010}(f ; X, A)$, then we can homotope $f$ to $g:(X, A) \rightarrow$ $(X, A)$ with $N(f ; X, A)$ fixed points in $X$, of which $\sum_{A_{j} \in \mathcal{A}-\mathcal{S}_{0}} N\left(f_{j}\right)-k_{1}$ lie in $\operatorname{Int}(A), N(f ; X-A)+k_{2}$ lie in $X-A$, and therefore $\sum_{A_{j} \in \mathcal{S}_{0}} N\left(f_{j}\right)+$ $k_{1}+N_{1010}(f ; X, A)-k_{2}$ lie on $\operatorname{Bd}(A)$.

P r o of. We construct a finite subset $P$ in $X$ with $|P|=N(f ; X, A)$ step by step as follows.

Let $\mathcal{F}_{1}^{*} \subseteq \mathcal{F}_{1}$ be the set of common fixed point classes of $f$ which do not assume their indices in $A$ and do not contain any essential fixed point classes of $f_{j}: A_{j} \rightarrow A_{j}$ for an $A_{j} \in \mathcal{S}_{0}$. Then $\left|\mathcal{F}_{1}^{*}\right|=\widetilde{n}(f ; X, A)-\widetilde{n}\left(f ; X, \mathcal{S}_{0}\right)$. For each $\mathbf{F} \in \mathcal{F}_{1}^{*}$, there is an essential fixed point class $\mathbf{F}_{j}$ of $f_{j}: A_{j} \rightarrow A_{j}$ with $\operatorname{Int}\left(A_{j}\right) \neq \emptyset$ such that $\mathbf{F}_{j} \subseteq \mathbf{F}$. We choose a point $p$ on $\operatorname{Bd}\left(A_{j}\right)$. After making this choice for all fixed point classes in $\mathcal{F}_{1}^{*}$, we get a set $P_{1} \subseteq \operatorname{Bd}\left(\mathcal{A}-\mathcal{S}_{0}\right)$ with $\left|P_{1}\right|=\widetilde{n}(f ; X, A)-\widetilde{n}\left(f ; X, \mathcal{S}_{0}\right)$.

Since $\widetilde{n}(f ; X, A)-\widetilde{n}\left(f ; X, \mathcal{S}_{0}\right) \leq k_{1} \leq \sum_{A_{j} \in \mathcal{A}-\mathcal{S}_{0}} N\left(f_{j}\right)$, we can add $k_{1}-\widetilde{n}(f ; X, A)+\widetilde{n}\left(f ; X, \mathcal{S}_{0}\right)$ new points on $\operatorname{Bd}\left(\mathcal{A}-\mathcal{S}_{0}\right)$ to $P_{1}$, and then we get a finite set $P_{2}$ such that $\left|P_{2} \cap \operatorname{Bd}\left(A_{j}\right)\right| \leq N\left(f_{j}\right)$ for each $A_{j} \in \mathcal{A}-\mathcal{S}_{0}$.

Adding $N\left(f_{j}\right)-\left|P_{2} \cap A_{j}\right|$ new points in $\operatorname{Int}\left(A_{j}\right)$ for each $A_{j} \in \mathcal{A}-\mathcal{S}_{0}$, and $N\left(f_{j}\right)$ new points in $A_{j}$ for each $A_{j} \in \mathcal{S}_{0}$, we get a finite set $P_{3}$ such that 
(1) $P_{3} \subseteq A$,

(2) $\left|P_{3} \cap A_{j}\right|=N\left(f_{j}\right)$ for each $A \in \mathcal{A}$,

(3) $\sum_{A_{j} \in \mathcal{A}-\mathcal{S}_{0}}\left|P_{3} \cap \operatorname{Bd}\left(A_{j}\right)\right|=k_{1}$.

Thus, for any $\mathcal{S} \subseteq \mathcal{A}$,

$$
\begin{aligned}
\left|P_{3} \cap \operatorname{Int}(\mathcal{S})\right|= & \left|P_{3} \cap \operatorname{Int}\left(\mathcal{S}-\mathcal{S}_{0}\right)\right|=\left|P_{3} \cap\left(\mathcal{S}-\mathcal{S}_{0}\right)\right|-\left|P_{3} \cap \operatorname{Bd}\left(\mathcal{S}-\mathcal{S}_{0}\right)\right| \\
\leq & \sum_{A_{j} \in \mathcal{S}-\mathcal{S}_{0}} N\left(f_{j}\right) \\
& -\mid\left\{\mathbf{F} \in \mathcal{F}_{1}^{*} \mid \mathbf{F} \text { is labelled by a point in } A_{j} \in \mathcal{S}-\mathcal{S}_{0}\right\} \mid \\
\leq & \sum_{A_{j} \in \mathcal{S}-\mathcal{S}_{0}} N\left(f_{j}\right)-\left(\widetilde{n}(f ; X, A)-\widetilde{n}\left(f ; X, \mathcal{S}-\mathcal{S}_{0}\right)\right) \\
\leq & \sum_{A_{j} \in \mathcal{S}} N\left(f_{j}\right)-\widetilde{n}(f ; X, A)+\widetilde{n}(f ; X, \mathcal{S}) .
\end{aligned}
$$

Since $0 \leq k_{2} \leq N_{1010}(f ; X, A)=\left|\mathcal{F}_{2}\right|$, we can pick a subset $\mathcal{F}_{2}^{*}$ of $\mathcal{F}_{2}$ with $\left|\mathcal{F}_{2}^{*}\right|=N_{1010}(f ; X, A)-k_{2}$. For each $\mathbf{F} \in \mathcal{F}_{2}^{*}$, there is therefore an inessential fixed point class $\mathbf{F}_{j}$ of $f_{j}: A_{j} \rightarrow A_{j}$ with $\mathbf{F}_{j} \subseteq \mathbf{F}$. We choose a new point $p$ on $\operatorname{Bd}\left(A_{j}\right)$ as the labelling point of $\mathbf{F}$. Add these $N_{1010}(f ; X, A)-k_{2}$ labelling points to $P_{3}$ to get a finite set $P_{4}$ in $A$. For any $\mathcal{S} \subseteq \mathcal{A}$, we have

$$
\begin{aligned}
\left|P_{4} \cap \mathcal{S}\right| & =\left|P_{3} \cap \mathcal{S}\right|+\left|\left(P_{4}-P_{3}\right) \cap \mathcal{S}\right| \\
& =\sum_{A_{j} \in \mathcal{S}} N\left(f_{j}\right)+\mid\left\{\mathbf{F} \in \mathcal{F}_{2}^{*} \mid \mathbf{F} \text { is labelled by a point in } A_{j} \in \mathcal{S}\right\} \mid \\
& \leq \sum_{A_{j} \in \mathcal{S}} N\left(f_{j}\right)+\left|\left\{\mathbf{F} \in \mathcal{F}_{2}^{*} \mid \mathbf{F} \in i_{\mathrm{FPC}}\left(\operatorname{FPC}\left(f_{j}\right)\right), A_{j} \in \mathcal{S}\right\}\right| \\
& \leq \sum_{A_{j} \in \mathcal{S}} N\left(f_{j}\right)+\left|\left\{\mathbf{F} \in \mathcal{F}_{2} \mid \mathbf{F} \in i_{\mathrm{FPC}}\left(\operatorname{FPC}\left(f_{j}\right)\right), A_{j} \in \mathcal{S}\right\}\right| \\
& =\sum_{A_{j} \in \mathcal{S}} N\left(f_{j}\right)+n(f ; X, \mathcal{S}) .
\end{aligned}
$$

Add $N(f ; X-A)+k_{2}$ distinct points in $X-A$ to $P_{4}$ to get a finite set $P$ with

$$
\begin{aligned}
|P| & =\left|P_{4}\right|+N(f ; X-A)+k_{2} \\
& =\left|P_{3}\right|+\left(N_{1010}(f ; X, A)-k_{2}\right)+N(f ; X-A)+k_{2} \\
& =N(\bar{f})+N_{1010}(f ; X, A)+N(f ; X-A)=N(f ; X, A) .
\end{aligned}
$$

Notice that $\left|P \cap A_{j}\right|=\left|P_{4} \cap A_{j}\right| \geq\left|P_{3} \cap A_{j}\right|=N\left(f_{j}\right)$ for each component $A_{j}$ of $A$, and $P \cap \operatorname{Int}(\mathcal{S})=P_{3} \cap \operatorname{Int}(\mathcal{S}), P \cap \mathcal{S}=P_{4} \cap \mathcal{S}$ for any $\mathcal{S} \subseteq \mathcal{A}$. Thus, the set $P$ satisfies the conditions of Theorem 4.1, and then we get the conclusion. 
Setting $k_{1}=\widetilde{n}(f ; X, A)-\widetilde{n}\left(f ; X, \mathcal{S}_{0}\right)$ and $k_{2}=0$ in the theorem above, we have

TheOREM 5.8. Let $(X, A)$ be a pair of compact polyhedra satisfying the conditions in the theorem above. Then any map $f:(X, A) \rightarrow(X, A)$ is homotopic to a map $g:(X, A) \rightarrow(X, A)$ with $N(f ; X, A)$ fixed points in $X$, of which $\sum_{A_{j} \in \mathcal{A}-\mathcal{S}_{0}} N\left(f_{j}\right)-\widetilde{n}(f ; X, A)+\widetilde{n}\left(f ; X, \mathcal{S}_{0}\right)$ lie in $\operatorname{Int}(A)$, $N(f ; X-A)$ lie in $X-A$, and therefore $\sum_{A_{j} \in \mathcal{S}_{0}} N\left(f_{j}\right)+N_{1010}(f ; X, A)+$ $\widetilde{n}(f ; X, A)-\widetilde{n}\left(f ; X, \mathcal{S}_{0}\right)$ lie on $\operatorname{Bd}(A)$. So there are $\widetilde{N}^{\prime}(f ; X, A)$ fixed points in $\mathrm{Cl}(X-A)$.

Acknowledgements. I would like to thank Professor Robert F. Brown of UCLA for sending me the preprint of [7], which enabled me to get a better expression for the relative Nielsen numbers by using bipartite graphs.

\section{References}

[1] R. F. Brown, The Lefschetz Fixed Point Theorem, Scott and Foresman, Glenview, IL, 1971.

[2] P. Hall, On representatives of subsets, J. London Math. Soc. 10 (1935), 26-30.

[3] B. Jiang, On the least number of fixed points, Amer. J. Math. 102 (1980), 749-763.

[4] - Lectures on Nielsen Fixed Point Theory, Contemp. Math. 14, Amer. Math. Soc., Providence, RI, 1983.

[5] H. Schirmer, A relative Nielsen number, Pacific J. Math. 122 (1986), 459-473.

[6] - On the location of fixed points on pairs of spaces, Topology Appl. 30 (1988), $253-266$.

[7] P. Wolfenden, Fixed point sets of deformations of polyhedra with local cut points, Trans. Amer. Math. Soc. 350 (1998), 2457-2471.

[8] X. Z. Zhao, A relative Nielsen number for the complement, in: Topological Fixed Point Theory and Applications, B. Jiang (ed.), Lecture Notes in Math. 1411, Springer, Berlin, 1989, 189-199.

[9] —, Basic relative Nielsen numbers, in: Topology-Hawaii, World Sci., Singapore, $1992,215-222$.

Department of Mathematics Capital Normal University

Beijing 100037, P.R. China

E-mail: zhaoxve@mail.cnu.edu.cn

Received 9 June 1998 